\title{
Information theory in ecosystems
}

\author{
Arthur Michalowski
}

Published online: 25 March 2011

(C) The Author(s) 2011. This article is published with open access at Springerlink.com

\begin{abstract}
Steering of management processes in compliance with resources of information in ecosystems, is one of the bases of knowledge-based economics which is conditioned by the achievement of sustainable development. The aim of this paper is to present the problems that became the subject of the research on the information theory in ecosystems as the domain of sustainable development economics. The analysis of information within ecosystem ought to be perceived as the methodology of scientific conduct, which is supportive of management processes in the conditions of stable and sustainable development. In such perspective, this analysis lies in the recognition of what is the structure of phenomena, processes and streams of information within ecosystems and in the cybernetic system: ecosystem-economy. The analysis comprises also those research activities that serve explanation whether certain management processes came into being or will be realized in accordance with the principles of sustainable development. Thus, the results of information within ecosystem may prove useful not only for making decisions concerning the courses and ways of acting for the benefit of preserving ecosystems, but they may also enable control, evaluation and greater economic efficiency and effectiveness of steering sustainable development.
\end{abstract}

Keywords System · Information - Environment - Sustainable development . Sustainable development economics

JEL Classification Q01 Q Q57 · B41

\footnotetext{
A. Michalowski $(\varangle)$

Chair of Spatial Economy and Economic Policy, Department of International Relations and National Security, Stanislaw Staszic College of Public Administration in Bialystok, Dojlidy Fabryczne 26, 15-555 Bialystok, Poland e-mail: amichalowski@wsap.bialystok.pl
} 


\section{Introduction}

Toffler industrialization wave has contributed to the unprecedented scientific and technological advancement as well as to the development of many world regions and to the high living standard of 1/5 of world population. However, at the same time there have been observed unprecedented threats related to the environment at all organization levels of nature. Although the wave has not affected all the world regions so far, it needs be replaced by another wave-known as the wave of knowledge-based economy. Owing to knowledge it is now possible to overcome the threats caused by industrial advancement, i.e. global warming, lowering level of ground waters on the continents, decline of biological diversity on a global scale, and others. It appears that thanks to knowledge and its consilience (Wilson 1998) it will be possible not only to preserve the natural conditions in which people live and to make progress, but it will also contribute to the advancement of production and services that do not pose any threat to ecosystems and enable achievement of considerably higher living standard.

It needs emphasizing that steering of management processes in compliance with resources of information in ecosystems is one of the bases of knowledge-based economics which is conditioned by the achievement of sustainable development. This paper constitutes the attempt to present the problems related to the research on information theory in ecosystems as the domain of sustainable development economics.

\section{Information theory}

Etymologically, the term 'information' comes from the Latin word informatio, which signifies 'representation, explanation, notification'. The concept informatio in ancient Latin also meant giving form and shaping, as well as instructing, teaching and forming mind. Since 'information theory' was formulated and the 'general theory of communication systems' was presented there have been formed various expressions related to the term 'information'. These expressions are used in various domains, among others in systems theory, cybernetics, natural sciences or management sciences. Most frequently into consideration one takes the concept of information which is perceived as the data concerning processes and phenomena. Moreover, the role of information is emphasized in the process of decision-making. Owing to the fact that the term 'information' is not clearly defined it is differently in particular domains of science.

In 1948 the mathematician and engineer Shannon published the monograph The mathematical theory of communication, which constituted a part of his research conducted at Bell Systems. In the subsequent year this monograph was published at the University of Illinois. However, it became supplemented with the comments by Weaver who coordinated the research on analogue calculators during World War II. The works by Shannon enabled the development of the hypothesis based on mathematical theory of communication which played a crucial role in transforming the models dominating in pure sciences and in transferring them into the domain of communication. Shannon (1948) defined information as the expected message selected from the set of possible messages. The greater is the set of possible messages, the more information is present in the given message, and the amount of this is measured using the binary 
algorithm. Mathematical formula of information is based on the formula of mathematical expected value, which was earlier known as mathematical hope. On the basis of the aforementioned assumptions there has been defined a sign (symbol), information carrier, code, channel, transformation (translation) of code and message. In English the word 'communication' means connection, i.e. transport of information, not transport of matter. Emergence of the concept 'information' may not be separated from biological research. Following the formulation of mathematical theory of communication by Shannon (1948), the language of information and codes began to be present in biology. In 1943 Schrödinger used it for explaining the significance of the models of unitary development included in chromosomes. Since that time the organizing power of information analogy has become part of the greatest discoveries in this domain: discovery of DNA by the American scientist Avery in 1944 as the basis for heredity; indication of the double spiral structure by two scientists: Crick from Great Britain and Watson from the United States of America; and also the research on genetic code conducted by the French Nobel Prize Winners: Jacob, Lwoff and Monod. While formulating his theory, Shannon availed himself of the achievements concerning biology of the nervous system. And adversely, mathematical theory of communication served the specialists in molecular biology as the framework for those concepts that are necessary for grasping biological specificity or peculiarity of an individual (Michalowski 2007).

Information is chiefly defined as:

(1) the essence taken from the outside world in the process of adjusting us to it and adjusting our senses to it;

(2) message obtained by man thanks to observance or mental activity, and then transmitted in the arrangement: sender-receiver;

(3) properties of signal or message that are related to reducing the non-specific character of situation or to its further development;

(4) essence taken from the outside world, which expands knowledge or reduces lack of knowledge the decision maker, uncertainty and non-specific character of decision-making situation;

(5) essence of special importance of something, for somebody and owing to something, expressed using linguistic and/or non-linguistic signs;

(6) reflection of what exists in material sense (the so-called relation of reflection), and factor defining to a certain degree the form (shape) of future things and phenomena, the so-called relation of realization;

(7) data concerning economic processes and phenomena, used in the decisionmaking process.

The aforementioned definitions imply that information can be considered both in a wide and narrow perspective. The wide perspective of information means that this is not only message about something, but also every decision, ban, suggestion or recommendation. This message can be transmitted not only in the arrangement: man-man, but also in other systems in which the functions of a sender and a receiver may be performed by living creatures, machines or other objects. Here one can observe a special type of information-i.e. metainformation, i.e. information about information. Whereas in the narrow perspective information implies the message received by a 
receiver through observation or mental activity, which is conveyed in the following arrangement: sender (man)-receiver (man). In this case, the concept 'information' is reduced in terms of both its essence and point of reference. However, in each case the structure of information is composed of essence, carrier, symbol by means of which information is recorded and the way of transmitting it.

An interesting study of information was conducted by Berman (1991). The analysis of information conducted by this scientist points at four meanings of it:

(1) thing-information is the product of a certain process with its performer (source of information) and user (receiver), it may be subject to such operations as producing, storing, sending, transforming, exchanging, purchasing and selling;

(2) measurable value - there are situations in which transferred information includes either too much or too little information. Therefore, one of the problems encountered at the level of quantity information theory is the optimum of information in communication processes;

(3) potential - it is related to the quantity aspect of information included in information and it is associated with the ability to cause a certain change of thing, i.e. to reduce or to eliminate uncertainty of the receiver in relation to the states considered by this receiver and selected among the possible ones (such approach plays a particular role in the process of decision-making);

(4) change-importance of it is related to the role of information in shaping attitudes and behaviors, i.e. it is closely linked with communication (Berman 1991).

In the literature devoted to information theory and information systems one can distinguish various types of information. The most important among them include:

(1) information about phenomena - created by data concerning systems; this concerns chiefly social, economic and ecological phenomena and systems which are external in relation to the receiver or user of information;

(2) information for decision making - this regards any type of information, regardless of its essence or form, that is used by the decision maker for making decision;

(3) steering information - this regards any decisions, orders and directives having impact on social, economic and ecological phenomena and processes;

(4) information that constitutes resources of the system - this is information that is in social, economic and ecological systems and is indispensable for their proper functioning; this information is usually organized in the form of subsystems that function as integral elements of systems; information that is created and is present there is the effect of the decisions made and constitutes the instrument of its realization;

(5) information having consequences - this regards any information, regardless of its essence or form, the emergence, change or lack of which in social, economic or ecological system has certain consequences.

Thus, information is the factor that reduces the lack of precision and has impact on the system's functioning and on the realization of its various functions. Change of the state of receiver, which was caused by information, takes place in varied ways:

(1) directly - through its reference to the intentional change, in the form of recommendation, instruction or warning; 
(2) indirectly - through making reference to the state which is the subject being recognized and described; this is connected with raising the level of knowledge and with creating bases for rational action; thus, information is related to communication which may be perceived as a special type of informing oriented at evoking a certain change (Czekaj 2000).

Most authors share the opinion that full explanation of the essence of information is possible on the level of information and in it into consideration should be taken its basic aspects: quantity and quality and also semantic and pragmatic aspects .

The concept 'information theory' is used in scientific and professional literature predominantly in three aspects:

(1) information theory - the theory of sending a message at a distance using technical methods; development of modern techniques and telecommunication systems required the creation of theoretical bases of data transmission;

(2) information theory - the theory of coding, the section of applied mathematics comprised by general cybernetics; this trend came into being on the ground of applying mathematical statistics in communication; information theory regards the bases of coding information, noises and interferences in the process of conveying information;

(3) information theory - the theory of information systems which lies in the analysis of all aspects of information processes; it is based on the assumption that there are certain general information processes that can be formalized and are common for the following systems: biological, social, economic and technical.

The last among the aforementioned aspects concerns among others the following issues:

(1) analysis and assessment of the properties of information as such, for instance its quality, value and usefulness;

(2) problems and methods of transforming information, especially analysis of disturbances in the processes of transformation (transforming, transmitting and storing information);

(3) problems and methods of reflecting information in various codes, informational aspects of codes and well as relations between languages.

The attempts to comprise the issue of information in theoretical systems were made in the 1940s, when dynamic growth of telecommunication technologies required sound theoretical bases. The 1950s and 1960s are known as the period of time marked by the development of information theories within cybernetics as the general theory of steering. At this time there were created many elaborations that shaped conceptual apparatus and methodological approaches to the analysis and synthesis of information and also to the information processes and systems. These theoretical concepts are known as information theories.

There is no singular information theory, but there are distinguished many theoretical concepts and approaches that in total enable analysis and synthesis of information processes and systems. Some elaborations played a crucial role in shaping the conceptual apparatus used for the analysis of information phenomena. However, presently 
these are seldom used although they contributed to development of knowledge and understanding of information processes. In this particular elaboration there will be presented solely the most essential elements of selected information theories that ought to be used in analyses of information in ecosystem. These are:

(1) quantity information theory;

(2) semantic information theory;

(3) pragmatic information theory;

(4) quality information theory (Olenski 1997).

In the modeling of ecosystem it is syntactic approach that appears as the most developed one. The bases of syntactic information theory stem from mathematic communication theory. According to many scientists, in this approach information is a universal property of matter and energy - it can be identified with any forms of diversity (Richling and Solon 2002). Resources of information are among the basic internal resources of ecosystems (ecological systems), apart from material and energetic resources.

\section{Information in ecosystems}

System is a theoretical concept that expresses complexity of phenomena. Systems can be recognized, steered and developed through modeling and making operations on models. With the purpose to construct a model of system one needs to define its assumptions as the point of reference. Above all, it is required to specify the aims and levels of organization (self-organization) of the modeled reality. In the next stage there should be designed variables used for construction of a model. Modeling is seldom expressed in a single set of operations. In practice, it is a process that is defined as a modeling path.

In modeling and analysis of systems there are two typical approaches: black box method and white box method, which ought to be perceived as complementary methods. The former method is based on the definition of a repertory of inputs and outputs of system that can be interpreted as a repertory of arguments and values of the transfer function. It may be treated as a simple mathematical concept without introduction of the time factor (time function). In case of recognizing mathematic formula that transforms arguments of transfer function into its values it is possible to specify the structure of system and to use efficiently the white box method-it can be modeled through identification and description of system parameters.

The concept of ecosystem was introduced as early as in the 1930s. At the beginning of the 1940s there was formed a postulate to study trophic dependencies in ecosystems using bioenergetic methods. International enthusiasm in relation to such research in the 1960s and 1970s resulted in a great number of publications. However, there is still lack of a coherent theory of ecosystem. Knowledge presented in books usually has a descriptive character (Weiner 2005).

In this elaboration there was adopted the following definition of ecosystem: It is a discretional fragment of biosphere in which a group of organisms realizes the processes of transforming chemical links in accordance with thermodynamics laws, in 
the conditions of closed circulation of matter, energy and information (Michalowski 2007, p. 41).

In accordance with the information theory, the structure of ecosystems is composed of its elements and links between them. The basic elements of ecosystems are specimens of particular species. These specimens create populations that can be transformed into larger groups. These can be treated as subsystems of ecosystems. Informational links are based on various ways of conveying information in ecosystems using the parameter of environment. Simultaneously, it is a signal transmitted between ecosystem elements. In the situation when a signal is transmitted from one element to all the others one can assume that it behaves like an entirety and displays a maximal coherence. On the basis of the aforementioned assumptions the following definition of information in ecosystems (information within ecosystems) can be adopted: material and energy streams that are found in ecosystems and enable transmission of information; and also as the existence of information streams. Information streams are divided into two types. In the first type information is conveyed together with its carrier, without transformation of it into a special form. In another one information is transformed prior to conveying of it (for instance sound signals and electric impulses). Effect of information streams does not lead to weakening and decline of resources of information in ecosystems (Michalowski 2007, p. 57).

Thermodynamic premises imply that the increasing level of information within system is related to its reduced entropy. This always takes place at the cost of its close surrounding. In an ecosystem there are physical barriers making unconstrained displacement of information impossible. They ensure its efficient functioning. The condition of their existence is changeless supply of energy characterized by high degree of ordering because these structures are dissipative. They are functioning in the realm of animate world and at the border of biotic and abiotic elements of ecosystems.

The functioning of ecosystems in the light of information theory is based on transmission channels of information that are observed. It can be an ecosystem as the entirety or its particular elements. Simultaneously they are transmitters of signals. The receivers of conveyed information are individuals that further on collect, transform and transmit it to other individuals - they communicate.

When compared with human standards, the number of signals used for transmission of information (communication) via other species appears to be very limited. In order to define quantity measures appropriate for this generalization, it is required to define a signal as any activity of transmitting information from one individual to another one, regardless of whether it could perform any other functions. Communication between animals takes place through demonstrations which, during evolution, became specialized in transfer of information. Demonstration is a signal that was changed in terms of reinforcing its signal function. Small diversification of signals in communication between animals is in opposition to the seemingly limitless output of human language. However, there are certain differences between man and other organisms. Paralinguistic signals of every human culture are comparable as regards a number of them to manifestations of animals. Human language is formed by arranging sounds and has increasing hierarchy of words and sentences characterized by redundancy that is satisfactory for making the distinction between them. 
Although the number of demonstrations recorded by environmentalists amounts only to several dozens per species, the real number of transmissions is much larger. In the simplest systems the demonstration may have only one meaning. However, in case of certain invertebrates and in most systems of vertebrates the amount of information transmitted by a single signal can increase thanks to reinforcing mechanisms. A signal may be graduated, linked with other signals simultaneously or in various sequences. Its sense is changing, depending on the environmental context. A special form of complex signals is metacommunication, i.e. communication concerning meaning of other communication acts. Many best organized communication systems among social insects include resources of information that can be transmitted not by one specimen to another one, but by one group to another one. This phenomenon is known as collective communication (Wilson 1975).

It is relatively simple to imagine the analysis of information transmission (communication), but it is much harder to make it. It is divided into three main parts:

(1) description of evolutionary or cultural origin of information;

(2) setting of transmission channel-from neurophysical process that initiates signaling activity, through mechanisms thanks to which signal is transmitted, conducted, received and interpreted.

(3) settlement of transmission function-its importance for communicating individuals and role in ecosystem functioning.

While comparing communication between closely related species it is possible to find the connections between evolution levels leading to the creation of complex communication systems. Every evolutionary change that reinforces communication functions is defined as semantization. The processes of semantization may regard answers, i.e. sensorial apparatus and behavior of species change in order to ensure more adaptive reaction that already exists and does not undergo transformations. The vast majority of well-known cases of semantic transformations are related to ritualization-evolutionary process as a result of which a model becomes more effective as a signal (Krebs and Davies 1993). In extreme cases a model is modified so considerably in relation to its initial form that it is impossible to recreate the history of its evolution. Ritualization and its consequences determine evolution of communication systems during which signals are created almost in every biological process. One may assume that species evolve towards a group of possibilities that maximize informative or/and energetic efficiency. It is a justified activity to analyze sensorial channels as if there was rivalry between them as regards transfer of information. Main transmission channels are composed of: chemical communication, aural communication, visual communication, tactile communication and electric communication.

The processes of communication forming, learning and forming social systems were found also in further groups of organisms, which has led to considerable differences in specialization and complexity. In this respect, four groups of animals can be distinguished: colonial invertebrates, social insects, mammals and people. The basic features of social life of each of these groups are specific for it. Although the aforementioned number of taxonomic groups leads from more primitive forms to the more advanced ones, the main properties of social organization, including closeness, 
altruism and inclination to cooperation, become less intensive. More advanced structure of particular organisms entails smaller proneness to social evolution.

\section{Information theory in ecosystems versus sustainable development economics}

While analyzing the interactions between the environment (ecosystems) and the system of global economy one needs to remember that not all pairs of systems can be created using informative function. Compatibility of two diverse communication systems or steering are defined as interface. It takes place when code of one system may be received and decoded by another system. With the purpose to withhold communication or steering it is necessary to partly close or change the interface. If the interface is entirely cut off, the communication or steering ceases. The problem of interface as universal communication between the environment and the system of economy is of particular importance for civilization because it decides upon its durability. Information exists only for the societies that have interface, i.e. are capable of decoding, understanding and using information within ecosystems. Communication and steering set into motion the entire environment enabling chemical reactions, metabolism, growth of living creatures and correlation between life in ecological, social and economic systems.

If systems have common interface, they can be linked with one another at one level or by means of hierarchy. As the one-level aggregate can serve the chain of elementary systems described by:

(1) number of elements in a certain system;

(2) sequence of elements in the chain;

(3) reversal (direction) of information function.

One-level aggregate is a form of direct steering. If the chain has both weaker and stronger elements, the chain works at the level of the weakest link. Therefore, in practice there are designed double (parallel) chains, which reinforces reliability.

Greater complexity is achieved by designing hierarchical systems-every system of lower level becomes an element of higher level system. This way of achieving complexity is universal in biosphere, and used by both nature and civilization. Every living creature is a hierarchical system. Every higher level in hierarchy presents higher informational quality.

If hierarchical systems cooperate or compete, one can observe steering that is either above or below a certain dimension. Steering above dimension is observed when steering level represents a higher complexity than the steered one. Steering below level is always efficient, and also impossible to be observed by the steered one, it is usually named manipulation. This type of steering is used among others in politics and marketing. Steering above level is known as inefficient, as the definition of the attempt to steer a more organized system by less organized one implies. Steering below level at times simply leads to catastrophe or collapse of the system.

Identification of the effect of steering creates feedback, which in cybernetics also has such name. Identification is defined as performance by feedback the criterion of identification. If this criterion is not fulfilled, information is rejected as it is extraneous 
to the system. System's ability to identify feedback is known as observability. Observability is indispensable for efficient steering however, it has certain limitations:

(1) steering subsystem may not identify itself;

(2) higher level in hierarchical system is not identifiable for the lower level;

(3) in the surrounding there are certain unobservable fields.

Observability can be either supported by system memory or not. In the former case there is mnemonic observability, in the latter case-unmnemonic observability.

Every feedback in open system has double content:

(1) concerning own steering subsystem;

(2) concerning answers of system surrounding.

The surrounding of system is seldom passive. The effect of steering disturbs the surrounding which can react in the form of processes of mutual steering. Thus, feedback comprises information about both our internal and external world. Feedback concerning the consequence of steering may be either certain or uncertain. If it is certain, one can state that the set of steering consequences is determined: static or dynamic. Here observability is ensured through circulation of signal that expresses either success in fulfilling a certain identification criterion (1) or not (0). Observability of this type requires solely a binary code that is composed of two signs: 1 and 0 (yes and no). Binary code supplies information concerning only steering cycle. If this criterion is fulfilled, the cycle is perceived as completed; if not-the cycle begins once again.

In this elaboration it has been assumed that the basic initial division of environmental system regards division into systems that are reasons of informative influences on other systems subjected to these influences (ecosystems). Systems having influences constitute realized undertakings and management processes in the conditions of sustainable development. Between the isolated systems there takes place transmission of data through inputs and outputs of systems.

So far economic theory has not handled the problem of information transmission between ecosystems and economy. The environment and its ecosystems altogether with the system of economy create cybernetic system. Apart from feedback, its properties include analysis of cause and effect processes. This ought to comprise natural, social, economic, sociological and cultural problems. Its purpose ought to be the formation of new models of sustainable development. Lack or inadequate knowledge of cause and effect relations is still the source of negative consequences found in ecosystems. This is also related to the fact that few environmental and economic problems are still analyzed via systemic perception of the environment.

Introduction of the issue of economic importance of information within ecosystem in management in the conditions of sustainable development is related to compliance with the premises ensuing from the thermodynamics of physical systems (theory of entropy) and from the dynamic balance of the macrosystem environmentsociety-economy. The first group of premises implies that some economic laws and dependencies lying at the bases of economic models are in the opposition to thermodynamic laws. Within the limits of the second group of premises there is statement in accordance with which dynamics of macrosystem depends on the scale of a phenomenon, and also that coevolutionary reactions do not have a linear character-i.e. 
small adaptation changes or relatively small disturbances can be the reason of great transformations. It contributes to the obligation to introduce the issues of information in ecosystems into economic theory and to treat them on equal terms as other variables having impact on development processes. In sustainable development it is required to emphasize relativity of this concept (Rogall 2008). This term ought to be defined for a certain scale of activity in time and space because sustainable development on the scale of continent or region may have entirely different character than on a local scale.

In many theoretical publications there is attempt to define threshold values of ecological variables that are characteristic for various time and space scales and after exceeding of which ecosystems begin to behave in an unpredictable way. There are many evidences showing that there are functional discontinuities (bifurcations) in those systems as the effect of gradual and slow increase in anthropogenic influences in time perspective. Occurrence of those discontinuities results from the fact that in management processes one cannot observe information coming from the inside of ecosystems (information within ecosystem) into the system of society and economy.

The aforementioned grasp of information transmissions from the inside of ecosystems into the system of society and economy, as well as receiving information from those systems into ecosystems and their elements have impact on the usage of information theory within ecosystem in desigining sustainable development economics, especially the processes of environmental management (Michalowski 2007, 2009). Presently, environmental economics is based chiefly on internalizing external costs, the basis of which is the concept of optimal Pigou tax and Coase's theorem. Proper functioning of both concepts in practice requires reliable ways and methods of estimating ecological benefits and losses that would take into consideration information within ecosystem that enters the system of society and economy. Owing to this obligation there is necessity to modify the system of methods of estimating benefits and losses in ecosystems. According to the Author, the usage of economic estimation in relation to ecosystems is presently limited due to lack of complete usage of economic estimation within ecosystem. Instead, economists focus on identification of various types of values of environmental goods, and to a smaller degree they are concerned with the attempts to analyze parameters of particular elements of ecosystems and streams of economic information ensuing from them.

In steering of socio-economic sustainable development into consideration are taken the principles adopted in the Earth Charter at the Earth Summit in Rio de Janeiro. They refer to four orders: environmental, economic, social and institutional-political.

Among 27 principles of the Charter as many as 18 refer to environmental order. Implementation of them is intended to contribute to introducing sustainable development into existence. The principles of sustainable development were defined also in several other European and national documents. On the basis of the analysis of a set of these principles Borys (2005) has drawn the following conclusions:

(1) most principles focus on two pillars of development: maintenance of equality among generations as regards access to environmental resources and maintaining efficiency of the environment;

(2) many pro-environmental principles emphasize integrity of all orders; 
(3) some sets of principles are considerably different than others as regards restrictive protection of ecosystems and their resources;

(4) in general, principles are satisfactory as the criterion of selecting the indicators specifying the degree of achievement or changes in environmental order.

In accordance with the aforementioned conclusions Borys (2005) adopts environmental indicators in relation to all levels of steering sustainable development: international, national, regional and local. Review of those indicators makes it possible to say that there is necessity to supplement indicators related with economic information within ecosystem.

For the needs of systemic steering of sustainable development there ought to be adopted such indicators the characteristic properties of which would reflect the real changes in ecosystems with certain precision that is required for economically effective steering of sustainable development. In practice of steering development, such indicators ought to be grouped into three sets of indicators: physical, chemical and biological (Michalowski 2007). As the source of data for those indicators should serve scientific research and also both knowledge and experience of practitioners dealing with environmental management, i.e. among others foresters, farmers, biologists-protectioners and managers.

\section{Concluding remarks}

In the times of knowledge-based economy and information society there occur more frequent remarks concerning the necessity to manage information and knowledge. Management of knowledge becomes a new domain of science that is characterized by very diverse roots. It still does not have entirely developed methodology. Some authors concentrate on information systems. Others lay emphasis on human aspect of knowledge generation and circulation, and reduce the issue to the level of policy concerning personnel. However, knowledge management has interdisciplinary character, and its cycle is based on discovering knowledge and using it. Thus the final subject of knowledge management becomes strive for the ideal of organization and multiplication of intellectual capital. In such approach knowledge is perceived as the key factor that conditions socio-economic development.

So far in management systems the knowledge that was shaped through analysis of streams of information within ecosystem was taken into consideration to a small degree. Introduction of it into the cycle of knowledge management in organizations to a large degree would increase efficiency of steering socio-economic sustainable development and shaping its orders.

The traditional model of environmental indicators of sustainable development is concentrated mainly on the indicators regarding the supply flows of matter and energy in ecosystems. Therefore, it is indispensable to extend environmental indicators so that they become indicators of information within ecosystem, the analysis of which is one of the basic conditionings of durable and sustainable management in the conditions of knowledge-based economy.

The analysis of information within ecosystem ought to be perceived as the methodology of scientific conduct that supports management processes in the conditions 
of stable and sustainable development. In such perspective it lies in recognizing the structure of phenomena, processes and streams of information inside ecosystems and in cybernetic arrangement of ecosystem-economy. It comprises those research activities that aim at explanation of whether certain management processes existed or they will be realized in accordance with the principles of stable and sustainable development. Thus, the results of analyses concerning information within ecosystem may serve not only making decisions regarding directions and types of activities serving maintenance of ecosystems, but they also enable control, assessment and increase in economic efficiency and effectiveness of steering sustainable development.

Information within ecosystem in the system of knowledge-based economy and in the conditions of sustainable development requires further theoretical work, including work concerning its recognition, estimation of its value, usefulness, quality and importance in economic terms.

Acknowledgments The author thanks Janet Landa and anonymous reviewers for comments on earlier versions of this manuscript.

Open Access This article is distributed under the terms of the Creative Commons Attribution Noncommercial License which permits any noncommercial use, distribution, and reproduction in any medium, provided the original author(s) and source are credited.

\section{References}

Berman, C. (1991). Information and aspects of communication. Scientific Issues, 3(4), 426-434.

Borys, T. (Ed.). (2005). Sustainable development indicators. Warsaw: Publ. Ekonomia i Srodowisko.

Czekaj, J. (2000). Methods of information management in a company. Cracow: Publication of Cracow University of Economics.

Krebs, J. R., \& Davies, N. B. (1993). An introduction to behavioural ecology. Oxford: Blackwell Science Ltd.

Michalowski, A. (2007). Information in ecosystems. Bialystok: Ekopress.

Michalowski, A. (2009). Economic activity and natural processes. Bialystok: College of Public Administration.

Olenski, J. (1997). Information standards in economy. Warsaw: Publication of the University of Warsaw.

Richling, A., \& Solon, J. (2002). Landscape ecology. Warsaw: PWN.

Rogall, H. (2008). Ecological economics. An introduction. Wiesbaden: VS Verlag.

Shannon, C. E. (1948). A mathematical theory of communication. Bell System Technical Journal, 27, $379-423$.

Weiner, J. (2005). Life and evolution of the biosphere. Warsaw: PWN.

Wilson, E. O. (1975). Sociobiology: The new synthesis. Cambridge: Belknap Press of Harvard University Press.

Wilson, E. O. (1998). Consilience. The unity of knowledge. New York: A. A. Knopf. 